\title{
Final Technical Report :
}

\section{Title: "Purification, growth, fabrication and characterization of wide bandgap materials" SUBMITTED TO THE DEPARTMENT OF ENERGY \\ Grant No. DE-FG08-95NV11893 \\ Fisk No. 140-340 \\ by

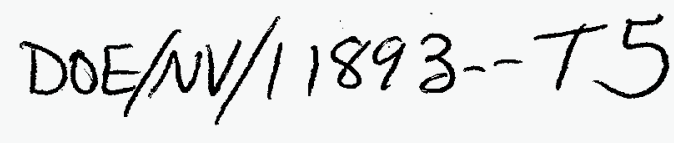

\section{Kuo-Tong Chen, Henry Chen and Arnold Burger \\ Department of Physics, Fisk University \\ Nashville, Tennessee 37208}

\section{Summary}

Wide bandgap semiconductor single crystals, such as heavy metal halide compounds, have been grown by physical vapor transport and Bridgman methods. Zone-refining and vacuum sublimation techniques were used to purify and adjust the stoichiometric composition of the starting material, and were proven to be effective. Several spectroscopic, microscopic and thermodynamic analytical techniques were employed to investigate the optical, electrical and structural properties of crystals. These results revealed information regarding micro- and macroscopic defects, impurities and modifications resulting from source material, growth process, post-growth treatment and device fabrication. Crystal growth and processing conditions have been correlated with this information and were optimized to achieve the purest and highest quality materials for practical device applications. Future works will involve optimization of material purification and crystal growth processes to produce high purity and low defect crystals, development of sensitive material characterization tools allowing a better understanding of defects formation and their correlation with processing conditions. Developments in bulk crystal growth research for detector devices in the Center for Photonic Materials and Devices since its establishment have been reviewed. Purification processes and single crystal growth systems employing physical vapor transport and Bridgman methods were assembled and used to produce high purity and superior quality wide bandgap materials based on heavy metal halides semiconductors. Comprehensive material characterization techniques have been employed to reveal the optical, electrical and thermodynamic properties of crystals, and the results were used to establish improved material processing procedures. 


\section{DISCLAMMER}

This report was prepared as an account of work sponsored by an agency of the United States Government. Neither the United States Government nor any agency thereof, nor any of their employees, makes any warranty, express or implied, or assumes any legal liability or responsibility for the accuracy, completeness, or usefulness of any information, apparatus, product, or process disclosed, or represents that its use would not infringe privately owned rights. Reference herein to any specific commercial product, process, or service by trade name, trademark, manufacturer, or otherwise does not necessarily constitute or imply its endorsement, recommendation, or favoring by the United States Government or any agency thereof. The views and opinions of authors expressed herein do not necessarily state or reflect those of the United States Government or any agency thereof. 


\section{DISCLAIMER}

Portions of this document may be illegible electronic image products. Images are produced from the best available original document. 


\section{Introduction}

The Materials Science and Application Group (MSAG) in the Fisk University Center for Photonic Materials and Devices (CPMD) has investigated the fabrication and evaluation of radiation detectors based on wide bandgap compound semiconductors (heavy metal halides). These materials not only have great potential in medical, industrial and environmental applications, but also in space exploration, as $\mathrm{X}$-ray and gamma ray spectrometers and imaging arrays. In this paper we report recent developments in materials processing and characterization and device fabrication.

\section{Material Purification and Crystal Growth}

Typically, starting materials which may purchased from commercial vendors with nominal purity of $99.9999 \%$ or synthesized from pure elements by stoichiometric weight, need to be further purified and/or adjusted to stoichiometric composition before crystal growth and device fabrication. In our laboratory, zone-refining and vacuum sublimation are two standard processes to achieve this goal. Zone-refining was first introduced in 1952, and has been successfully implemented by us to purify elements, such as $\mathrm{Se}$ and $\mathrm{Te}$, and heavy metal halides, such as $\mathrm{HgI}_{2}, \mathrm{PbI}_{2}$ and $\mathrm{BiI}_{3}$. The characteristic effect of zone-refining is to accumulate impurities at the ends of an ingot, thus leaving pure material in the central section. Figure 1 shows the distribution of impurity concentrations of $\mathrm{Mg}, \mathrm{Ag}, \mathrm{Cu}$ and $\mathrm{Cr}$ along a zone-refined ingot of $\mathrm{HgI}_{2}$ (ICP-MS analysis performed at Sandia National Labs, Livermore, Dr. Ralph James' research group). In this particular case it is clear that most impurities accumulated at one end (1-4) leaving the other section to be the purest part of the ingot. A similar purification effect has been observed for $\mathrm{PbI}_{2}$. Vacuum sublimation is a routine method used in our laboratory to purify starting materials from impurities having higher (under dynamic vacuum) or lower (closed tube) vapor pressures. Especially, baking the starting material under a dynamic pumping before sealing off the crystal growth ampoule plays an important role in 


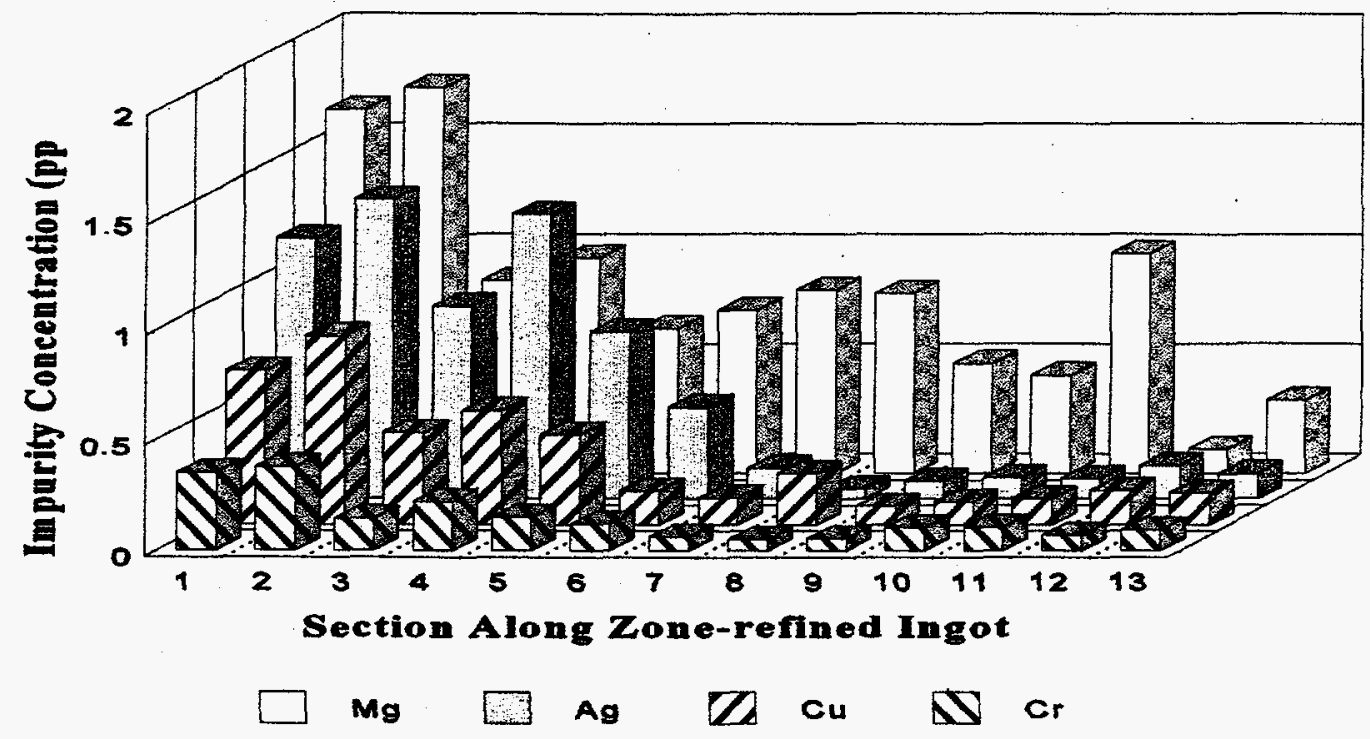

Figure 1. Impurity concentration distribution along zone-refined $\mathrm{HgI}_{2}$.

correcting the deviation from stoichiometry of the material.

Two crystal growth methods frequently used in our laboratory are Physical Vapor Transport (PVT) and Bridgman methods. PVT method is the crystal growth under vapor - solid equilibrium conditions. The temperature of the starting material (powder form) is higher than the nucleation/crystal growth region. This imposed temperature gradient leads to a diffusiveconvective flow resulting in a net mass transport of vapor species towards the crystal growth site. The vapor species may consist of molecules of the material itself, such as $\mathrm{PbI}_{2}$ (solid) $\rightarrow \mathrm{PbI}_{2}$ (vapor), or dissociated into its separate constituents, such as $\mathrm{CdTe}$ (solid) $\rightarrow \mathrm{Cd}$ (vapor) $+1 / 2 \mathrm{Te}_{2}$ (vapor), and residual gases. The reverse process occurs when vapor species nucleate and then continue to condense on the crystal growth interface at a rate of 3-5 mm/day. 


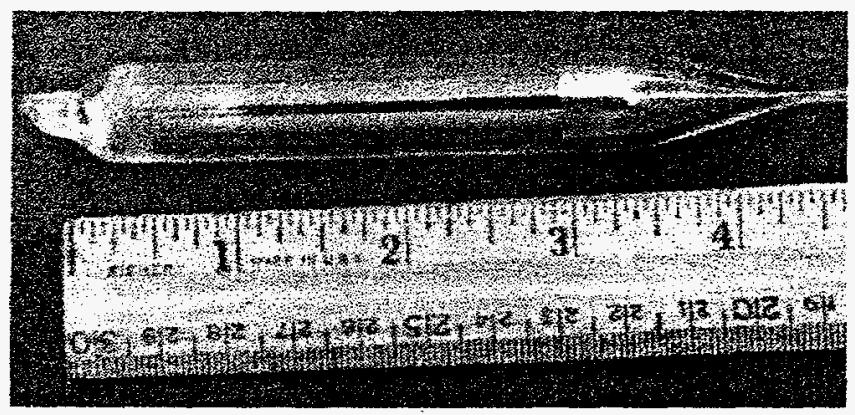

Bridgman grown $\mathrm{PbI}_{2}$

Figure 2. As-grown lead iodide single crystals in the growth ampoule.

The Bridgman growth method is basically a controlled freezing process taking place under liquid solid equilibrium conditions. The growth also takes place under a temperature gradient, and the mechanism is to produce a single nucleus from which a single crystal will propagate and grow. This is achieved by allowing the solid - liquid interface to move slowly (5-50 $\mathrm{mm} /$ day) until the whole molten charge is solidified. $\mathrm{A} \mathrm{PbI}_{2}$ single crystal is shown in Figure 2. Compared to other growth methods, Bridgman method is considered to be a rather simple crystal growth method, but several limitations still exist. The Bridgman method can not be applied to a material system which decomposes before it melts, systems having components with high vapor pressure, and materials exhibiting destructive solid - solid phase transformations which will compromise the crystalline quality on cooling the crystal at the end of the growth run. $\mathrm{HgI}_{2}$ is a typical a material which is not suitable for Bridgman growth. It has high vapor pressure and undergoes a solid - solid phase transition from $\alpha \rightarrow \beta$ at $130^{\circ} \mathrm{C}$ before its melting temperature at $258^{\circ} \mathrm{C}$. Therefore $\mathrm{HgI}_{2}$ can only be grown only by $\mathrm{PVT}$, while $\mathrm{PbI}_{2}$, lacking such a phase transition can also be grown by Bridgman method.

\section{Materials Characterization}

Several characterization methods based on spectroscopies, microscopies and thermal analysis techniques have also been employed to investigate and reveal the optical, electrical, structural and thermodynamic properties of the grown crystals.

A) Leakage current measurements of improved vapor grown $\mathrm{BiI}_{3}$ crystals.

I-V curves of two triple sublimations samples from ORNL (Dr. Lynn Boatner) were measured (set \#1). First sample (small) had a thickness of $1.2 \mathrm{~mm}$ and the larger sample had a thickness of 1.4 $\mathrm{mm}$. Pt contacts with an area of $0.196 \mathrm{~cm}^{2}$ were sputtered on each side of the crystals without any 
prior surface treatment. The results show the following: The small sample had an apparent resistivity of $10^{6} \mathrm{ohm} . \mathrm{cm}$. The contacts were removed, the crystal was washed in methanol and contacts were applied again. The apparent resistivity increased to $10^{7} \mathrm{ohm} . \mathrm{cm}$. The same experiments were repeated for the large sample, except that the etchant used in this case was Br-methanol solution. In both cases the resistivity was $10^{9} \mathrm{ohm} . \mathrm{cm}$. Although both samples did not show any gamma ray response due to high leakage currents, these results represent an increase of three orders of magnitude in resistivity compared to $\mathrm{BiI}_{3}$ samples previously measured by us, including double sublimed $\mathrm{BiI}_{3}$. The results also show the need for proper surface preparation. Surface leakage effects may still be the dominant component in the I-V curves measured by us and there is need for more experiments to find the optimized etchant.

B) Contact mode Atomic Force Microscopy studies of $\mathrm{CdZnTe}$ crystals and detectors.

CdZnTe crystals obtained from Digirad and eV Products companies were studied related to their surface preparation during the fabrication of nuclear detectors. Several etchants were selected and tested. The best etching procedure, that minimizes the leakage currents of the detectors, was found to be a modified (by the addition of lactic acid) solution of bromine in methanol. The surface roughness measured by AFM was the lowest for this etchant. After appling the electric contacts and encapsulating the area of the contacts, we have developed a surface passivation treatment of the lateral faces of the detector. The treatment involves the oxidation of these surfaces in a $20 \%$ hydrogen peroxide $\left(\mathrm{H}_{2} \mathrm{O}_{2}\right)$ solution. Gold contacts were deposited using three different procedures: sputtering, electroless deposition and thermal evaporation. Platinum contacts were deposited using the sputtering technique. Current voltage measurements were performed on all samples and thermal evaporated gold were found to be less injective and therefore produce best signal-to-noise nuclear detectors. This findings were presented at the International SPIE meeting to be held in Denver, CO. and IEEE Nuclear Science Symposium to be held next November in Anaheim, CA.

C) Crystal growth of $\mathrm{PbI}_{2}$ material.

Zone refined lead iodide material was used in the growth of single crystals using the Brigdman technique. One graduate student and four undergraduates were involved in the growth and characterization of the crystals. The study shows the single crystals of lead iodide ( 2 inch long, 
half inch diameter) can be routinely grown from the melt. Differential Scanning Calorimetry DSC measurements do not show any significant deviations from the stoichiometry within the sensitivity range of the experiment. The fabrication of nuclear detectors from this material is underway.

D) Characterization of $\mathrm{PbI}_{2}$ single crystals

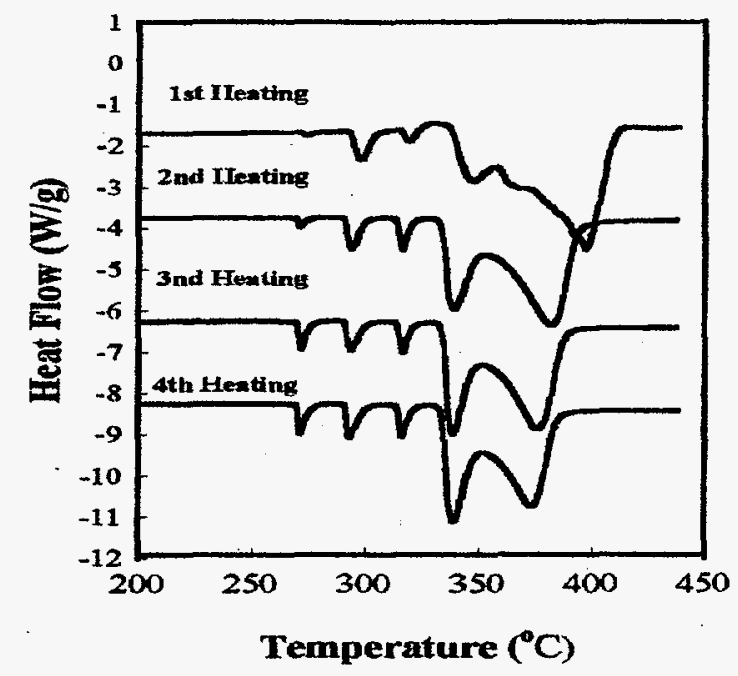

Lead iodide single crystals were characterized. Atomic Force Microscopy (AFM) studies have Figure 4. DSC thermograms of Bridgman grown $\mathrm{BiI}_{3}$.

revealed a highly superior morphology compared with bismuth iodide crystals. Except for cleavage steps no inclusions or other defects could be observed. One graduate student and four undergraduates were involved in the fabrication of nuclear detectors. Gold sputtered contacts have been applied to the opposite faces of a $1 \mathrm{~mm}$ thick lead iodide wafer. The resistivity of the material was measured and found to be in the $10^{12} \mathrm{ohm} \cdot \mathrm{cm}$ range. The nuclear detectors were tested and they show counting response.

E) Thermal analysis

Differential scanning calorimetry (DSC), has been employed for characterization of mechanical and thermodynamic properties such as phase transitions and melting temperatures, heat of fusion and impurity analysis of materials. Figure 4 shows the DSC thermograms of a Bridgman grown $\mathrm{BiI}_{3}$ crystal. While the melting temperature for stoichiometric $\mathrm{BiI}_{3}$ is $408^{\circ} \mathrm{C}$, the Bridgman grown crystals show a depression of melting temperature and some second phases toward low temperature during the first heating run. These second phases were more developed during the second, third and fourth heating cycles, and at the same time the melting temperature has been depressed even more. These second phases were identified from temperature-composition phase diagrams as Bi-rich phases coexisting in a $\mathrm{Bil}_{3}$ solid solution. PVT grown $\mathrm{BiI}_{3}$ was also investigated by DSC, not shown here, and the thermograms showed no second phase with a melting temperature near $408^{\circ} \mathrm{C}$. These DSC results clearly show that PVT is the most suitable method for crystal growth of $\mathrm{BiI}_{3}$ and the melting process produced $\mathrm{Bi}$-rich second phases. 
F) Student training

Five undergraduate students have participated during two months of the summer of '96 in projects related to purification, growth, fabrication and characterization of wide bandgap semiconductor materials:

1) Ms. Catrice Simmons, worked on a vacuum technology project including vacuum deposition of thin films that are used in detector contact application

2) Ms. LaToya Suber and Mr. Kurt Hansen worked on a Differential Scanning Calorimetry project aimed at establishing the phase diagram of $\mathrm{Pb}$-rich lead iodide material

3) Ms. Serena Wilson and Mr. Jamie Henderson worked on purification and crystal growth of lead iodide material using the Bridgman method. 